\title{
Obtenção de nanofios de carbono a partir de copolímero de PAN eletrofiados para aplicação como supercapacitores
}

\author{
Obtaining carbon nanofibers \\ from electrospinning PAN copolymers \\ for application as supercapacitors
}

\author{
Hugo Moreira da Silva Costa ${ }^{1}$, Valdinei Euzébio Rodrigues ${ }^{1}$, \\ Jorge Tadao Matsushima ${ }^{1,2}$, Mauricio Ribeiro Baldan ${ }^{2}$, Mirabel Cerqueira Rezende ${ }^{3}$ \\ Rita de Cássia Mendonça Sales ${ }^{1}$
}

\footnotetext{
${ }^{1}$ Faculdade de Tecnologia de São José dos Campos - Professor Jessen Vidal, FATEC, Av. Cesare Mansueto Giulio Lattes, 1350, Eugênio de Melo, São José dos Campos, São Paulo, Brasil.

${ }^{2}$ Instituto Nacional de Pesquisas Espaciais, INPE-SJC, Av. dos Astronautas, 1758, São José dos Campos, São Paulo, Brasil.

${ }^{3}$ Universidade Federal do Estado de São Paulo, UNIFESP, Campus SJC, R. Talim, 330 - Vila Nair, São José dos Campos, São Paulo, Brasil.

e-mail: hugomoreiracosta@hotmail.com, valdinei.rodrigues@fatec.sp.gov.br, jorge.matsushima@ fatec.sp.gov.br, mauricio.baldan@inpe.br, mirabelcr@gmail.com, rita.sales@ fatec.sp.gov.br
}

\section{RESUMO}

Atualmente, existe uma demanda na busca por materiais com alta densidade de potência, longo ciclo de vida e baixo impacto ambiental que são em sua maioria utilizados para a produção de supercapacitores, sendo esses considerados fontes promissoras de energia para sistemas eletrônicos. Os tipos de materiais mais promissores para essa aplicação são os baseados em carbono, devido à sua flexibilidade, área superficial e boa estabilidade eletroquímica. Para a produção de materiais carbonosos, o principal precursor utilizado é a poliacrilonitrila (PAN) e suas variações a partir da adição de monômeros, como o acrilato de metila (MA). Geralmente, esses materiais são utilizados em formas de mantas, fios ou nanofibras produzidos a partir de processos de fiação e posteriormente carbonizados para gerar o material carbonáceo. Um dos processos de fiação que tem sido amplamente estudado para a obtenção de nanofibras de carbono é o processo de eletrofiação. Portanto, este trabalho apresenta os resultados obtidos do processo de carbonização de mantas eletrofiadas a partir de solução de PAN homopolímero (PANH) e PAN-co-acrilato de metila $6 \%$ (PA6MA) com dimetilformamida (DMF) com o intuito de obter material para uso em supercapacitores. Os polímeros utilizados foram analisados por DSC para a obtenção dos valores relacionados a faixa de ciclização, sendo possível observar uma melhor estabilidade térmica relacionada as amostras de PAN6MA com faixa de temperatura de $240-312{ }^{\circ} \mathrm{C}$ e temperatura máxima do pico exotérmico de $292{ }^{\circ} \mathrm{C}$. As mantas obtidas pelo processo de eletrofiação foram oxidadas a $235^{\circ} \mathrm{C}$ por $5 \mathrm{~min}$ e carbonizadas a $900{ }^{\circ} \mathrm{C}$ por $5 \mathrm{~min}$. As mantas obtidas, antes e após o processo de carbonização, foram analisadas por MEV apresentando fibras de superfície lisa, dispersas aleatoriamente e de dimensões nanométricas, sendo os valores de diâmetro aproximado de $219 \mathrm{~nm}$ para as nanofibras de carbono de PANH (NfcPANH) e $185 \mathrm{~nm}$ para as nanofibras de carbono de PAN6MA (NfcPAN6MA). As mantas carbonizadas foram analisadas pela técnica RAMAN e foi possível a obtenção de dados referentes a estrutura carbônica presente nas amostras, onde os valores obtidos demonstraram que as amostras de NfcPAN6MA apresentaram menor valor de grau de cristalinidade medida a partir da relação entre as bandas $\mathrm{D}$ e $\mathrm{G}$ conhecido como fator $\mathrm{I}_{\mathrm{D}} / \mathrm{I}_{\mathrm{G}}$, sendo o valor obtido igual a 1,06 para as NfcPNA6MA e 1,24 para as NfcPANH, isso demonstra uma maior presença de estruturas grafíticas cristalinas quando comparadas as amostras produzidas a partir da PANH. Para obtenção das características capacitivas foram realizados levantamento das curvas carga/ descarga por análise cronopotenciométrica, foi possível observar as melhores características relacionadas a capacitância específica e estabilidade de ciclagem em baixas correntes aplicadas para as amostras de PAN6MA com valores de capacitância específica de 270,9 F/g, densidade de energia de $30,0 \mathrm{Wh} / \mathrm{kg}$ e densidade de potência de $153,5 \mathrm{~W} / \mathrm{kg}$. Os resultados obtidos demonstraram que o 
material apresentou potencial para a aplicação proposta.

Palavras-chave: Eletrofiação, Carbonização, Supercapacitor, Copolímero PAN.

\section{ABSTRACT}

Currently, there is a demand in the search for materials with high power density, long life cycle and low environmental impact that are mostly used fot the supercapacitors production, which are considered promising energy power for electronic systems. The most promising types of materials for this application are those based on carbon, due to their flexibility, surface area and good electrochemical stability. For the production of carbonaceous materials, the main precursor used is polyacrylonitrile (PAN) and its variations from the addition of monomers, such as methyl acrylate (MA). Generally, these materials are used in forms of blankets or yarns produced from spinning processes and later carbonized to generate the carbonaceous material. One of the spinning processes that has been widely studied for obtaining carbon nanofibers is the electrospinning process. Therefore, this paper presents the results obtained from the carbonization of electrospun blankets manufactured from solutions of polyacrylonitrile homopolymer (PANH) and poly (acrylonitrile-co-6\% methyl acrylate) (PAN6MA) combined with dimethylformamide in order to obtain material for use in supercapacitors. The polymers used were analyzed by DSC to obtain the values related to cyclization, by that were possible to observe a better thermal stability related to the samples of PAN6MA, with a temperature range of $240-312{ }^{\circ} \mathrm{C}$ and maximum temperature of the exothermic peak of $292{ }^{\circ} \mathrm{C}$. The electrospun mats were oxidized at $235^{\circ} \mathrm{C}$ per 5 min and carbonized at $90^{\circ} \mathrm{C}$ per $5 \mathrm{~min}$. Both pre-carbonization and post-carbonization mats were analyzed by SEM, showing fibers with smooth surface, randomly dispersion and nanometric size, with values of diameter of approximately $219 \mathrm{~nm}$ for PANH carbon nanofibers (NfcPANH) and $185 \mathrm{~nm}$ for PAN6MA carbon nanofibers (NfcPAN6MA). The carbonized mats were analyzed by RAMAN technique and it was possible to obtain the data related to the carbon structure present in the samples, where the obtained values showed that the mats manufactured from PAN6MA presented the lower value of degree of crystallinity measured from the relationship between bands D and G known as factor $\mathrm{I}_{\mathrm{D}} / \mathrm{I}_{\mathrm{G}}$, the value obtained being equal to 1.06 for NfcPAN6MA and 1.24 for NfcPANH, this shows a higher concentration of crystalline graphitic structures when compared with the samples produced from PANH. To obtain the capacitive characteristic, the load/discharge curves were surveyed by chronopotentiometric analysis, where it was possible to observe the best characteristics related to the specific capacity and low current cycling stability applied to the PAN - co- 6\% methyl acrylate sample with specific capacity values of $270.9 \mathrm{~F} / \mathrm{g}$, energy density of $30.0 \mathrm{Wh} / \mathrm{kg}$ and power density of $153.5 \mathrm{~W} / \mathrm{kg}$. The results obtained demonstrated that the material presented potential for the proposed application.

Keywords: Electrospinning, Carbonization, Supercapacitor, PAN copolymers.

\section{INTRODUÇÃO}

Atualmente, a técnica mais barata e que tem sido explorada para a obtenção de fibras precursoras da fibra de carbono é a eletrofiação ou electrospinning. Esta é uma técnica eletrostática de fabricação versátil, simples e barata de produção de nano/micro fibras e que, ao longo dos últimos anos, tem sido profundamente investigada e aprimorada para a produção de materiais nanoestruturados para diversas aplicações, tais como: engenharia de tecidos (área médica), filtros, vestuário de proteção, sistemas catalíticos e material eletrodo e aplicações óticas, permitindo o desenvolvimento de produtos de elevada qualidade a um preço reduzido [1-6].

Em um processo típico de eletrofiação um potencial elétrico é aplicado entre uma gotícula de solução polimérica expelida em velocidade controlada por uma bomba de seringa e um coletor aterrado. Quando o campo elétrico rompe a tensão superficial da gotícula um jato de solução polimérica carregado é atraído para o coletor [7]. O solvente evapora de forma contínua e rápida enquanto o jato é esticado por repulsão eletrostática formando no coletor aterrado nanofibras contínuas solidificadas [8-11].

O processo de eletrofiação envolve diversos fatores que são classificados em parâmetros relacionados a solução, ao ambiente e ao processo em si. Os parâmetros relacionados a solução podem ser a concentração de polímero, volatilidade do solvente, peso molecular, viscosidade, tensão superficial e densidade de carga superficial. Para o ambiente os parâmetros envolvidos são umidade, temperatura e fluxo de ar. Já para os parâmetros de processo tem-se o potencial elétrico, taxa de fluxo de liberação da solução, tipos de coletores, diâmetro da capilaridade ejetora e distância entre o coletor e a capilaridade ejetora [12].

A poliacrilonitrila (PAN) é o polímero mais utilizado como precursor para a produção de nanofibras de carbono por eletrofiação [6, 13]. Atualmente, as fibras de PAN em dimensões nanométricas apresentaram propriedades superiores às microfibras e tem se destacado nas aplicações industriais. A PAN possui algumas vantagens sobre outros polímeros, principalmente como um precursor de materiais carbonosos, devido ao seu elevado 
rendimento de carbono e uma estrutura termicamente estável e extremamente orientada [13-15].

Com o intuito de modificar as propriedades da PAN homopolímero vários comonômeros tais como ácido acrílico (AA), ácido metacrílico (MAA), ácido itacônico (IA), acrilato de metila (MA) e metacrilato de metila (MMA) possuem registro de copolimerização com a acrilonitrila. O monômero de MA é geralmente usado industrialmente para a melhoria das propriedades de fiação por fusão de copolímeros de PAN, pois reduzem a interação entre os grupos nitrílicos, reduzem a energia de ativação, reduzem a formação de estruturas core-shell e aumentam a solubilidade [16].

Além disso, para a fabricação de fibras de carbono com resistência mecânica elevada foram utilizados exclusivamente precursores de copolímeros de PAN contendo de 0,5-8\% de comonômeros, pois esses tornam os copolímeros mais prontamente solúveis em solventes para fiação permitindo uma melhor orientação da cadeia molecular nas fibras precursoras e tornando as fibras estabilizadas e carbonizadas mais estruturalmente homogêneas [17].

Nanofibras de carbono, como outras nanoestruturas unidimensionais (1D) tais como nanofios, nanotubos e fios moleculares, estão recebendo cada vez mais atenção por causa de sua grande relação comprimento/diâmetro [18]. Isto é devido ao seu potencial aplicação em nanocompósitos [19], catálise a alta temperatura, modelo para nanotubos [20], filtros [21] baterias recarregáveis [22], supercapacitores [23, 24], aplicações de montagem em nanoeletrônica e fotônica [25].

Para que as nanofibras produzidas por eletrofiação sejam transformadas em nanofibras de carbono, essas devem passar pelos processos para a obtenção da fibra de carbono tradicional, que são: a estabilização, a carbonização e a ativação, caso a aplicação do material seja como eletrodo ou a estabilização, a carbonização e a grafitização, caso a aplicação do material seja estrutural [26, 27].

Portanto, o objetivo deste trabalho é a obtenção de nanofibras de carbono produzidas a partir de matériaprima elaborada pela indústria nacional: PAN homopolímero (PANH) e PAN-co-acrilato de metila 6\% (PAN6MA). As nanofibras foram obtidas através do processo de eletrofiação. Os polímeros recebidos foram analisados por calorimetria diferencial de varredura (DSC) para obtenção da faixa de temperatura de ciclização de cada polímero. As fibras poliméricas eletrofiadas foram analisadas por microscopia eletrônica de varredura (MEV) antes e após os processos de oxidação e carbonização para a análise morfológica. A partir das amostras carbonizadas foram realizadas análises Raman para obtenção da estrutura carbônica predominante (desordenada ou grafítica cristalina) seguido de análise cronopotenciométrica para obtenção das características capacitivas. E por fim, os resultados obtidos das características morfológicas e capacitivas da nanofibras de carbono são comparados para determinar se as nanofibras de carbono possuem aplicabilidade como supercapacitores.

\section{MATERIAIS E MÉTODOS}

\subsection{Materiais}

Para a produção das fibras poliméricas por eletrofiação foram preparadas soluções com concentração de $6 \% \mathrm{~m} / \mathrm{m}$ de PAN- homopolímero (PANH) em DMF (dimetilformamida/ 99,5\% de pureza/ Merck ${ }^{\circledR}$ ) e $6 \% \mathrm{~m} / \mathrm{m}$ de PAN-co-acrilato de metila $6 \%$ (PAN6MA) também em DMF. Tanto o DMF quanto os polímeros utilizados foram fornecidos pelo Instituto Granado de Tecnologia da Poliacriloanitrila (IGTPAN).

\subsection{Metodologia}

\subsubsection{Caracterização da matéria-prima}

Para caracterizar os polímeros assim como recebidos, foi realizada a análise DSC no Instituto Tecnológico de Aeronáutica utilizando-se de equipamento NETZSCH DSC $404 \mathrm{C}$. A análise foi realizada a partir da temperatura ambiente até a temperatura de $400^{\circ} \mathrm{C}$ com rampa de $5^{\circ} \mathrm{C} / \mathrm{min}$ em atmosfera de Hélio.

\subsubsection{Sistema de Eletrofiação}

As amostras obtidas foram fabricadas utilizando-se o sistema de eletrofiação pertencentes a UNIFESP-SJC. O conjunto de equipamentos que compõem o sistema consiste em seringa de vidro $(20 \mathrm{~mL})$ conectada a uma agulha de aço de tipo Hamilton, bomba de seringa modelo Legato 220 da KDS Scientific, fonte de alta tensão (0$30 \mathrm{kV}$ ) da marca Faísca e um coletor cilíndrico feito de alumínio com diâmetro e comprimento de $175 \mathrm{~mm}$ x $132 \mathrm{~mm}$, respectivamente. $\mathrm{O}$ coletor foi devidamente aterrado e conectado a um motor de $0,33 \mathrm{hp}$ modelo IP55 da WEG, capaz de fornecer rotações de até $3100 \mathrm{rpm}$. Estes elementos foram adequadamente dispostos em uma caixa de acrílico (1000 mm x $600 \mathrm{~mm}$ x $500 \mathrm{~mm})$, como mostrado na Figura 1. 


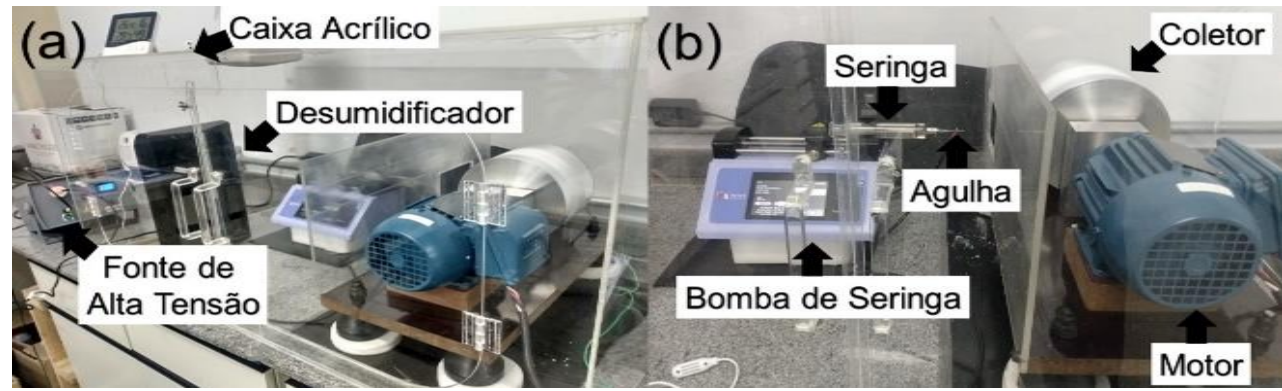

Figura 1: Sistema de Eletrofiação pertencente a UNIFESP-SJC: (a) Vista Isométrica e (b) Vista Frontal.

\subsubsection{Parâmetros definidos para os processos de eletrofiação}

Para a eletrofiação dos polímeros é necessário a dissolução desses em DMF (dimetilformamida), para tal foram preparadas soluções com concentrações de $6 \% \mathrm{~m} / \mathrm{m}$ de PANH e de PAN6MA em DMF As soluções descritas anteriormente foram preparadas por agitação em $55^{\circ} \mathrm{C} \pm 5{ }^{\circ} \mathrm{C}$ durante $1,5 \mathrm{~h}$ em agitador magnético da marca EVEN, modelo $\mathrm{HJ}-4$.

Os parâmetros utilizados no processo de eletrofiação foram tensão de $13,5 \mathrm{kV} \pm 1 \mathrm{kV}$, umidade entre 30 $45 \%$, taxa de infusão de $1,5 \mathrm{~mL} / \mathrm{h}$, distância entre a ponta da seringa e o coletor de $80 \mathrm{~mm}$ e rotação do coletor de $1000 \mathrm{rpm}$.

\subsubsection{Processo de oxidação e de carbonização das fibras obtidas por eletrofiação}

Após a obtenção das nanofibras poliméricas (NFP), essas foram oxidadas em estufa da marca Fanem, modelo Orion 515 , em $235^{\circ} \mathrm{C}$ por $5 \mathrm{~min}$ em atmosfera ambiente e em seguida carbonizadas em forno tubular EDG Equipamentos, modelo EDG10P-S em $900{ }^{\circ} \mathrm{C}$ por 5 min em atmosfera de argônio para a obtenção das nanofibras de carbono (NFC).

\subsubsection{Caracterização das fibras oxidadas e carbonizadas}

Tanto as NFC quanto as NFP foram analisadas via MEV com canhão de emissão de campo modelo SEM - FEG Quanta 650, auxiliado pelo software VegaTCx64 para observação da orientação e diâmetro das mesmas, visualização de possíveis poros superficiais, defeitos decorrentes do processo de eletrofiação e o impacto da carbonização nas NFP.

Para a caracterização do arranjo das cadeias carbônicas contidas nas fibras após os processos de oxidação e carbonização foi utilizada a técnica RAMAN utilizando-se de espectrômetro Raman Horiba modelo LabRam HR com comprimento de onda de $514 \mathrm{~nm}$.

Para obtenção das características eletroquímicas das amostras de NFC foram utilizados potenciostato AUTOLAB 302, modelo AUT84095, tipo PGSTAT302N, com auxílio de Booster AUTOLAB modelo BST7321, tipo BSTR10A, eletrodo de platina e solução contendo 2,0 mol/L de $\mathrm{H}_{2} \mathrm{SO}_{4}$, sendo os dados gerados pelo auxílio do software NOVA. A análise do comportamento eletroquímico foi feita por voltametria cíclica em uma faixa de potencial de $0-1 \mathrm{~V}$ em diferentes velocidades de varredura.

Para a análise da capacitância específica $\left(C_{\text {esp }}\right)$, densidade de potência $\left(d_{P}\right)$ e densidade de energia $\left(d_{E}\right)$ foram obtidas as curvas galvanostáticas de carga e descarga em uma faixa de potencial de 0-1 V e corrente de 0,5mA. A partir das curvas de carga/ descarga foram realizados cálculos da capacitância especifica de acordo com a Equação 1, aonde $C_{\text {esp }}$ é a capacitância especifica $(\mathrm{F} / \mathrm{g})$ i é a corrente aplicada $(0,5 \mathrm{~mA}), \Delta \mathrm{t}$ é o tempo para a descarga (s), $\Delta \mathrm{V}$ é a variação de potencial na descarga $(\mathrm{V})$ e $\mathrm{m}$ é a massa de material utilizada como eletrodo e previamente medida $(\mathrm{g})$.

$$
C_{e s p}=\frac{i \times \Delta t}{\Delta V \times m}
$$

A partir do resultado obtido para a capacitância especifica foi possível o cálculo da densidade de energia em $\mathrm{W} \cdot \mathrm{h} / \mathrm{kg}$ utilizando-se da Equação 2

$d_{E}=0,5 \times C_{e s p} \times\left(\Delta V^{2}\right)$

E com o resultado da densidade de energia foi realizado o cálculo da densidade de potência em W/kg de acordo com a Equação 3 


$$
d_{P}=\frac{d_{E}}{\Delta t}
$$

\section{RESULTADOS}

\subsection{Análise DSC}

A Figura 2 apresenta o termograma das amostras de PANH e PAN6MA obtidos na análise de DSC. As amostras foram aquecidas a taxa de $5{ }^{\circ} \mathrm{C} / \mathrm{min}$ sob atmosfera de He.

A partir dos resultados gerados pela análise de DSC foi possível a determinação das faixas de temperatura relacionados as reações de ciclização, representadas pelos picos exotérmicos, dos valores de entalpia e a determinação do valor máximo. Os valores obtidos são apresentados na Tabela 1. Estes valores são próximos àqueles encontrados na literatura para a PANH [28-32] e PAN6MA [28-30] como apresentado também está apresentado na Tabela 1. O desvio entre valores deste estudo e aos encontrados na literatura se dão devido as diferenças entre as taxas de aquecimentos empregadas nos ensaios, concentrações dos monômeros e atmosfera escolhidas para cada ensaio.

Sob a condição de He nenhuma reação oxidativa ocorreu durante esse processo, portanto, os picos exotérmicos das curvas de DSC das amostras de PANH e PAN6MA podem ser atribuídos às reações de ciclização. Como mostrado na Figura 2, existe apenas um pico exotérmico acentuado no homopolímero PAN. É sabido que na PANH, as reações de ciclização são iniciadas por um mecanismo de radicais livres, causando uma grande quantidade de calor ao mesmo tempo, o que resulta na quebra de cadeias moleculares liberando alguns tipos de componentes voláteis e com baixo desempenho. A curva DSC de PAN6MA possui a maior faixa de temperatura e pico mais amplo que o do PANH, mostrando que as reações de ciclização foram retardadas pela quebra da longa cadeia de acrilonitrila (AN) pelo monômero de acrilato de metila (MA) fazendo com que o processo ocorresse em temperatura mais baixa e as reações exotérmicas fossem diluídas, o que faz com que o copolímero de PAN6MA tenha uma reação de ciclização mais lenta e uma melhor estabilidade térmica, que é benéfico para a produção de fibras de carbono de alta performance [28, 29].

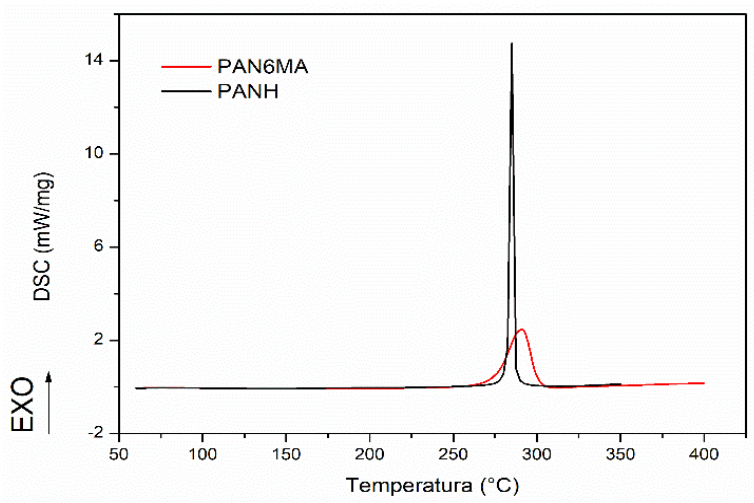

Figura 2: Termograma de DSC para os polímeros analisados como recebidos.

Tabela 1: Valores para a faixa de temperatura relacionados as reações de ciclização e de entalpia para os polímeros estudados.

\begin{tabular}{l|c|c|c|c}
\hline POLÍMERO & FONTE & FAIXA DE TEMPERATURA $\left({ }^{\circ} \mathbf{C}\right)$ & ENTALPIA $\Delta \mathbf{H}(\mathbf{J} / \mathbf{g})$ & T $_{\text {MÁx }}$ DOS PICOS $\left({ }^{\circ} \mathbf{C}\right)$ \\
\hline PANH & Autor & $275-295$ & 544,8 & 285 \\
\hline & Hao, et al. $[28]$ & $293,1-306,1$ & 527 & 299,6 \\
\hline & Bang, et al. $[29]$ & $257,63-X$ & - & 273,96 \\
\hline & Park, et al. $[30]$ & $239,4-X$ & - & 309,8 \\
\hline PAN6MA & Autor & $240-312$ & 559,2 & 292 \\
\hline & Hao, et al. $[28]$ & $267,7-307,1$ & 634 & 287,4 \\
\hline & Bang, et al. $[29]$ & $283-X-$ & - & 299,81 \\
\hline & Park, et al. $[30]$ & $263,3-X$ & - & 302,9 \\
\hline
\end{tabular}




\subsection{Análise morfológica das nanofibras antes e depois da carbonização}

As imagens de MEV das nanofibras poliméricas de PANH (NfPANH) e PAN6MA (NfPAN6MA) obtidas pelo processo de eletrofiação, são apresentadas na Figura 3. É possível observar pelas Figuras 3a e 3b que tanto a NfPANH quanto a NfPAN6MA apresentaram dispersão aleatória e superfície lisa, sem a apresentação de defeitos provenientes de processos de eletrofiação. Estes defeitos podem ser causados por vários fatores, dentre eles: i) união de fibras causada pela não evaporação do solvente devido a distância entre o jato da solução polimérica e coletor, ii) fibras ramificadas devido ao efeito a permissividade elétrica do coletor, iii) poros causados pelo efeito da umidade em um polímero hidrofóbico, iv) efeitos na forma de contas causada pelo efeito da densidade de cargas elétricas, entre outros [33].
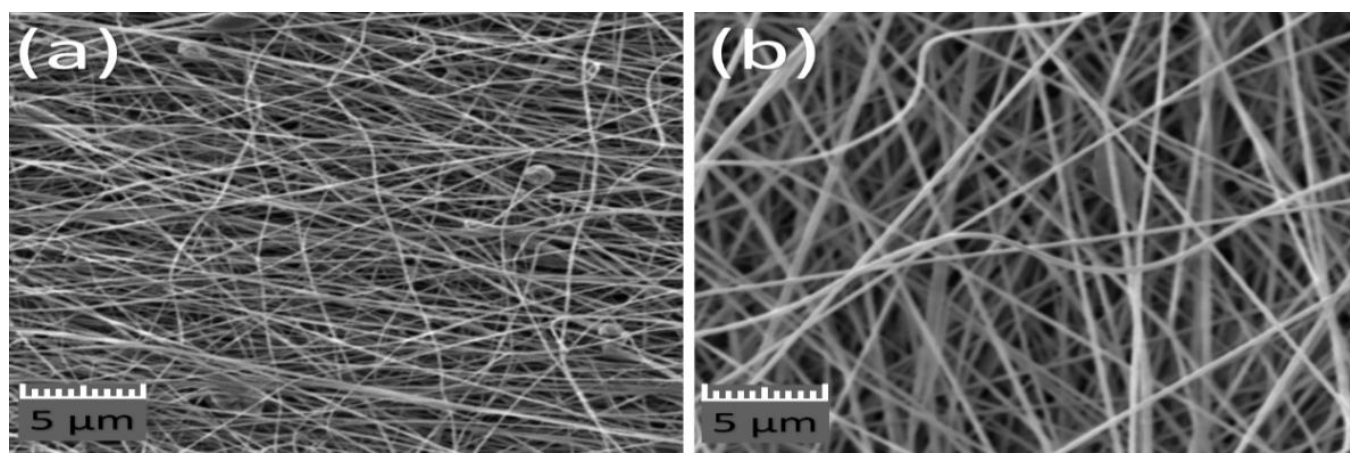

Figura 3: MEV das (a) NfPAN6MA e (b) NfPANH.

As imagens MEV das nanofibras de carbono de PANH (NfcPANH) e PAN6MA (NfcPAN6MA) são mostradas na Figura 4, onde é possível observar que os processos não interferiram na morfologia das fibras e que essas possuem diâmetros aproximados de $219 \mathrm{~nm}$ para as NfcPANH e de $185 \mathrm{~nm}$ para as NfcPAN6A. O cálculo do diâmetro médio das fibras foi feito a partir de 10 medições realizadas com o auxílio do software Image $\mathrm{J}$ em MEVs com magnificação de $10 \mathrm{kx}$ de ambas as amostras. Os resultados obtidos nas medições estão expostos na Tabela 2.
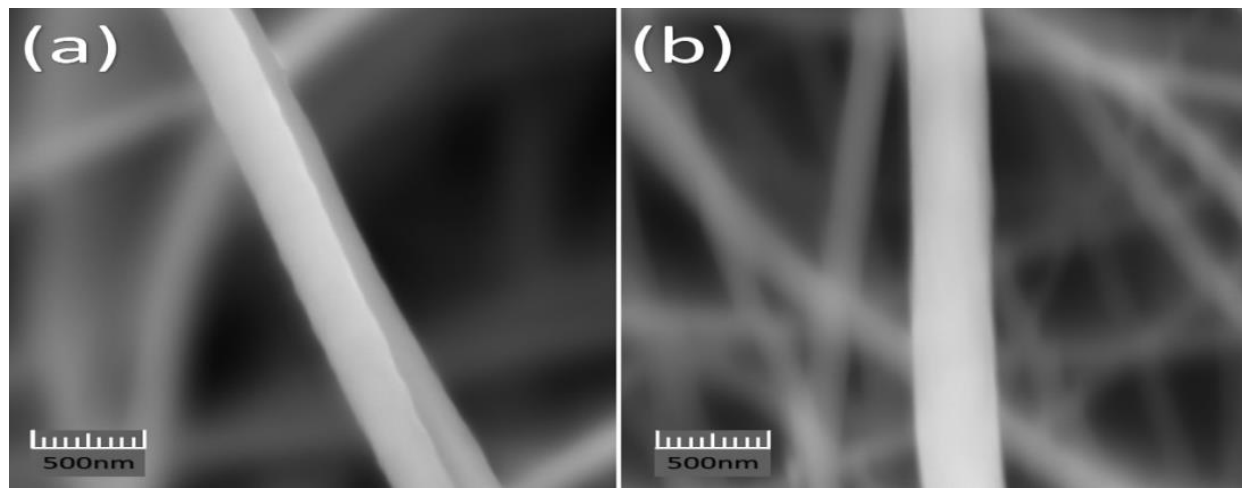

Figura 4: MEV das (a) NfcPAN6MA e (b) NfcPANH.

Tabela 2: Valores médios e desvio padrão dos diâmetros das NfcPANH e NfcPAN6MMA.

\begin{tabular}{l|c|c}
\hline AMOSTRA & MÉDIA (nm) & DESVIO PADRÃO \\
\hline NfcPANH & 219 & $\pm 0,024$ \\
\hline NfcPAN6A & 185 & $\pm 0,070$ \\
\hline
\end{tabular}

\subsection{Análise RAMAN}

Os resultados da análise Raman para as amostras NfcPANH e NfcPAN6MA estão apresentados na Figura 5. A partir da análise Raman é possível obter informações sobre a perfeição cristalina de materiais carbonáceos. 
Os dois picos apresentados nas análises de ambas as amostras são referentes as bandas D e G. A banda D localizada para a NfcPANH em aproximadamente $1360 \mathrm{~cm}^{-1}$ e para a NfcPAN6MA em aproximadamente $1356 \mathrm{~cm}^{-1}$ é relacionada a defeitos e estruturas desordenadas de carbono, enquanto a banda $\mathrm{G}$ localizada em aproximadamente $1584 \mathrm{~cm}^{-1}$ para a NfcPANH e $1587 \mathrm{~cm}^{-1}$ para a NfcPAN6MA é relacionada a estruturas grafíticas cristalinas [34-36]. A relação entre a área relacionada a banda $D\left(I_{D}\right)$ e a área relacionada a banda $G\left(I_{G}\right)$ é utilizada como um fator para medição do grau de cristalinidade de materiais carbonáceos $\left(\mathrm{I}_{\mathrm{D}} / \mathrm{I}_{\mathrm{G}}\right)$.

A melhor deconvolução das curvas para obtenção das áreas relacionadas a cada banda foram obtidas a partir de 4 bandas, sendo as duas primeiras relacionadas a banda D (D e D") e as outras duas relacionadas a banda $G\left(G\right.$ e $G$ '). Os valores obtidos para a largura das bandas $\left(\omega_{1 / 2}\right)$, número de onda $(\omega)$ e da relação $I_{D} / I_{G}$ são apresentados na Tabela 3.

Os valores da relação $\mathrm{I}_{\mathrm{D}} / \mathrm{I}_{\mathrm{G}}$ estão dentro do apresentado na literatura [34-38], sendo possível a observação de um menor valor para a NfcPAN6MA, demonstrando que o material possui mais estruturas grafíticas cristalinas em relação a amostra NfcPANH, o que pode estar relacionado a melhor estabilidade térmica observada pela análise de DSC contribuindo para um processo de oxidação mais eficiente. Segundo PARK, et al [30], o mecanismo de ciclização da PAN homopolímero ocorre através do mecanismo via radical livre, sendo o mesmo mecanismo presente na PAN-co-acrilato de metila, entretanto a presença do monômero de acrilato de metila age como um defeito ao quebrar a regularidade da longa cadeia de acrilonitrila. Portanto, o impacto do MA no copolímero desacelera a reação de ciclização, e para que a reação aconteça é necessário o consumo de mais energia provocando um controle da reação exotérmica. Devido a isso a PAN adicionada de MA possui maior estabilidade térmica do que a PAN Homopolímero.

Tabela 3: Valores de $\omega_{1 / 2}, \omega$ e $\mathrm{I}_{\mathrm{D}} / \mathrm{I}_{\mathrm{G}}$.

\begin{tabular}{|c|c|c|c|c|}
\hline AMOSTRAS & BANDAS & $\omega_{1 / 2}\left(\mathrm{~cm}^{-1}\right)$ & $\omega\left(\mathrm{cm}^{-1}\right)$ & $\mathrm{I}_{\mathrm{D}} / \mathrm{I}_{\mathrm{G}}$ \\
\hline \multirow{4}{*}{ NfcPANH } & $\mathrm{D}$ & 163,3 & 1356 & \multirow{4}{*}{1,24} \\
\hline & D” & 172,6 & 1208 & \\
\hline & $\mathrm{G}$ & 95,1 & 1597 & \\
\hline & G” & 120,7 & 1504 & \\
\hline \multirow{4}{*}{ NfcPAN6MA } & D” & 189,4 & 1352 & \multirow{4}{*}{1,06} \\
\hline & $\mathrm{D}$ & 143,1 & 1227, & \\
\hline & G” & 186,3 & 1600,27 & \\
\hline & $\mathrm{G}$ & 186,4 & 1533,22 & \\
\hline
\end{tabular}
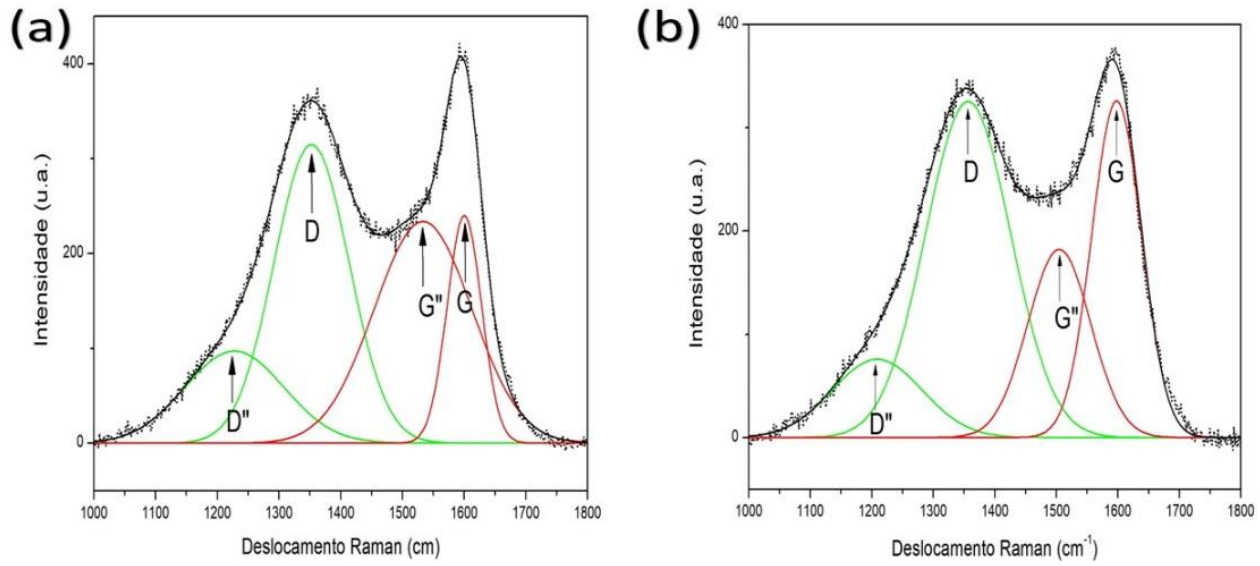

Figura 5: Resultados da análise RAMAN para (a) NfcPAN6MA e (b) NfcPANH. 


\subsection{Análise Cronopotenciométrica}

A Figura 6 apresenta os voltamogramas cíclicos das amostras na velocidade de varredura de $5 \mathrm{mVs}$ aonde é possível analisar que as mesmas apresentaram comportamento fora do esperado para um capacitor ideal aonde os voltamogramas cíclicos apresentam forma retangular, entretanto é possível observar que a amostra que mais se assemelhou com tal característica foi a NfcPAN6MA. A estrutura apresentada pela amostra NfcPANH mostrou que essa possui característica resistiva relacionada a inclinação da curva nos períodos de carga e descarga.

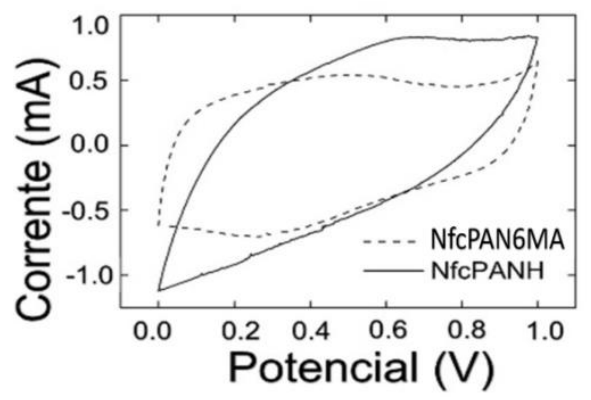

Figura 6: Voltamogramas cíclicos das amostras NfcPANH e NfcPAN6MA com velocidade de varredura de 5mVs.

Já as curvas de carga e descarga para as amostras são apresentadas na Figura 7 aonde foi possível os cálculos da $C_{\text {esp }}, d_{E}$, e $d_{P}$, de acordo com as Equações 1, 2 e 3 respectivamente. Os valores obtidos estão na Tabela 4.

Tabela 4: Valores de $C_{e s p}, d_{E}$ e $d_{P}$

\begin{tabular}{l|c|c|c}
\hline & $\mathbf{C}_{\text {esp }}(\mathbf{F} / \mathbf{g})$ & $\mathbf{d}_{\mathrm{E}}(\mathbf{W} \cdot \mathbf{h} / \mathbf{k g})$ & $\mathbf{d}_{\mathbf{P}}(\mathbf{W} / \mathbf{k g})$ \\
\hline NfcPANH & 270,9 & 30,0 & 153,5 \\
\hline NfcPAN6MA & 194,2 & 21,8 & 187,5 \\
\hline
\end{tabular}

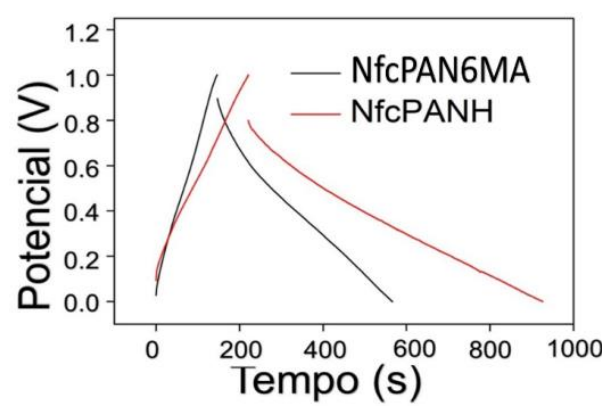

Figura 7: Curva Carga e Descarga para a NfcPANH e NfcPAN6MA.

\section{DISCUSSÃO}

Os valores de entalpia e temperatura de cristalização obtidos pela análise de DSC se aproximam aos resultados comprados aos encontrados na literatura. A análise térmica corroborou com os resultados obtidos pela análise de Raman, demonstrando que a NfcPAN6MA apresenta melhores condições relacionadas a estabilidade térmica devido a maior formação de estruturas grafíticas cristalinas em relação a amostra NfcPANH, indicado pela menor relação $\mathrm{I}_{\mathrm{D}} / \mathrm{I}_{\mathrm{G}}$ que contribui para um processo de oxidação mais eficiente.

A análise de MEV mostrou que as fibras obtidas apresentaram diâmetros nanométricos, superfície lisa e dispersão aleatória, não observando defeitos relacionados ao processo de eletrofiação. 
A partir dos resultados obtidos na análise cronopotenciométrica, foi possível observar maiores valores de $\mathrm{C}_{\text {esp }}$ e $\mathrm{d}_{\mathrm{E}}$, para a amostra NfcPANH $(270,90 \mathrm{~F} / \mathrm{g}$ e 30,0 Wh/kg) enquanto a amostra NfcPAN6MA (194,2 F/g e $21,8 \mathrm{Wh} / \mathrm{kg})$ apresentou maior valor para a $\mathrm{d}_{\mathrm{P}}(187,5 \mathrm{~W} / \mathrm{kg})$. Quando os valores são comparados com o diagrama de Ragone, apresentados na Figura 8, é possível notar que os valores de $d_{E}$ e $d_{P}$ para a amostra NfcPAN6MA $(30,0 \mathrm{Wh} / \mathrm{kg}$ e $187,5 \mathrm{~W} / \mathrm{kg})$ se aproximam mais da região considerada ideal para supercapacitores, entretanto os valores obtidos para ambas amostras se encontram na região considerada ideal para baterias. No gráfico de carga-descarga, apresentado na Figura 7, é notado uma queda abrupta do potencial no momento da descarga, em ambas as amostras, que está relacionada a resistência ôhmica da célula, sendo este valor de queda maior para a amostra NfcPANH corroborando o fato de que essa apresenta característica resistiva.

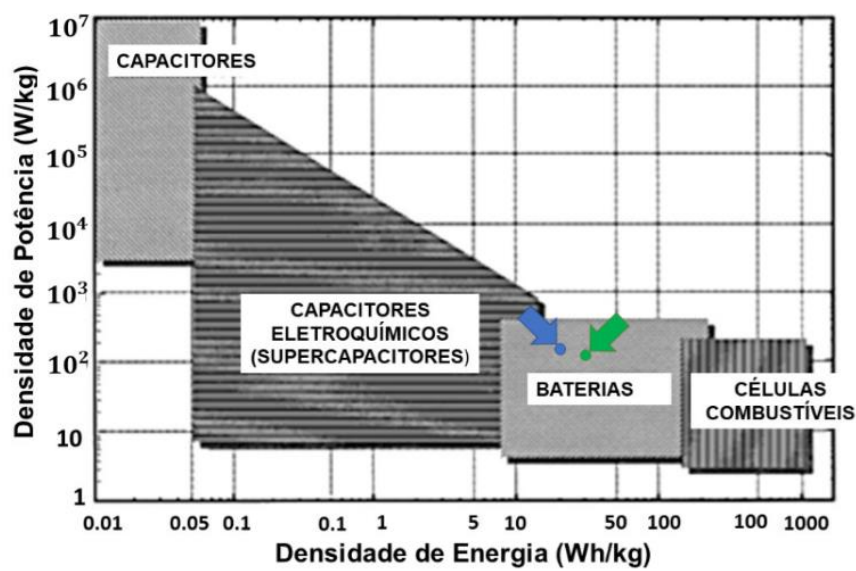

Figura 8: Diagrama de Ragone adaptado [39]: $(\square)$ Valores encontrados para NfcPAN6MA e $(\square)$ Valores encontrados para NfcPANH.

Os resultados obtidos validaram o uso dos materiais PANH e PAN6MA, produzidos pela indústria nacional, para a produção de material carbonoso. Este estudo possibilitou estabelecer a melhor rota de direcionamento de pesquisas para a geração de materiais para uso como supercapacitores.

\section{CONCLUSÕES}

A partir do resultado da análise de DSC foi possível concluir que o copolímero de acetato de metila contribuiu para a estabilidade térmica da PAN, o que pode ter levado ao menor valor de ID / IG obtido a partir da análise RAMAN para a NfcPAN6MA.

Pela análise de MEV foi possível concluir que as fibras eletrofiadas apresentaram diâmetro nanométrico, dispersão aleatória, superfície lisa e não apresentaram defeitos relacionados ao processo de eletrofiação, sendo possível também a observação de que os processos de oxidação e carbonização não geraram um impacto relevante na morfologia das fibras.

Os valores obtidos de capacitância específica, densidade de energia e de potência aproximaram-se dos valores esperados para aplicação como supercapacitores, mas não se enquadraram no quesito para tal, entretanto a NfcPAN6MA foi a que demonstrou o maior potencial para estudo futuro de variâncias nos processos de eletrofiação, oxidação e carbonização com o intuito da melhoria de suas qualidades capacitivas para aplicação como supercapacitores.

\section{AGRADECIMENTOS}

Os autores agradecem a UNIFESP São José dos Campos pela cessão do uso do sistema de eletrofiação, ao Instituto Granado de Tecnologia da Poliacrilonitrila (IGTPAN) pelo fornecimento da matéria prima para execução deste trabalho e ao INPE-SJC pela utilização dos fornos e equipamentos para análise das amostras e a bolsa de iniciação cientifica (processo $\mathrm{n}^{\text {o }} 152966 / 2019-6$ ). 


\section{BIBLIOGRAFIA}

[1] DIAS J.R., REIS I., BÁRTOLO P.J., "Potencial do Electrospinning para aplicações industriais Centro para o Desenvolvimento Rápido e Sustentado de Produto", Instituto Politécnico de Leiria, pp. 1-10, 2012.

[2] WU, M., WANG, Q., LI, K., et al., "Optmization of stabilization conditions for electrospun polyacrylonitrile nanofibers". DOI: 10.1016/j.polymdegradstab.2012.05.001 Polymer Degradation and Stability, v. 97, pp.15111519, Mai. 2012.

[3] QIN, X., YANG, E., LI, N., et al., "Effect of Different Salts on Electrospinning of Polyacrylonitrile (PAN) Polymer Solution". https://doi.org/10.1002/app.25498. Journal of Applied Polymer Science, v. 103, pp. 38653870, Jul. 2006.

[4] FENNESSEY, S.F., FARRIS, R.J. "Fabrication of aligned and molecularly oriented elesctrspun polyacrylonitrile nanofibers and the mechanical behavior of their twisted yarns".

https://doi.org/10.1016/j.polymer.2004.04.001. Polymer, v. 45, pp. 4217-4225, Dez. 2003.

[5] DZENIS, Y., "Spinning Continous Fibers for Nanotechnology". DOI: 10.1126/science.1099074. Science, v. 304, pp. 1917-1919, Set. 2012.

[6] NATARAJ, S.K., YANG, K.S., AMINABHAVI, T.M., "Polyacrylonitrile-based- nanofibers-A state-of-theart review". https://doi.org/10.1016/j.progpolymsci.2011.07.001. Progress in Polymer Science, v. 37, pp. 487513, Jul. 2011.

[7] GU, S.Y., REN, J., WU, Q.L., "Preparation and structures of electrospun PAN nanofibers as a precursor of carbon nanofibers". https://doi.org/10.1016/j.synthmet.2005.07.340. Synthetic Metals, v. 155, pp. 157-161, Mai. 2005.

[8] TRAN, C., KALRA, V., "Fabrication of porous carbon nanofibers with adjustable pore sizes as electrodes for supercapacitors". DOI:10.1016/j.jpowsour.2013.01.080. Journal of Power Sources, v. 235, pp. 289-296, Jan. 2013.

[9] OLIVEIRA, J.B., GUERRINI, L.M., OISHI, S.S., et al., "Carbon nanofibers obtained from electrospinning process". Materials Research Express, v. 5, pp. 1-11, Dez. 2017. https://iopscience.iop.org/article/10.1088/20531591/aaa467/meta

[10] PANAPOY, M., DANKEAW, A., KSAPABUTR, B., "Electrical Conductivity of PAN-based Carbon Nanofibers Prepared by Electrospinning Method". Thammasat International Journal of Science and Technology, v. 13, pp. 11-17, Nov. 2008.

[11] HUANG, Z., ZHANG, Y.-Z., KOTAKI, M., et al., "A review on polymer nanofibers by electrospinning and their applications in nanocomposites". https://doi.org/10.1016/S0266-3538(03)00178-7. Composites Science and Technology, v. 63, pp. 2223-2253, Jan. 2003.

[12] VAZ, B.S., COSTA, J.A.V., MORAIS, M.G., "Production of polymeric nanofibers with different conditions of the electrospinning process". e11847. Available from:

<http://www.scielo.br/scielo.php?script=sci_arttext\&pid=S1517-70762017000200514\&lng=en\&nrm=iso>.

Epub Jul., 2017. ISSN 1517-7076. http://dx.doi.org/10.1590/s1517-707620170002.0180. Matéria (Rio J.), v. 22, n.2 [cited 2021-01-28],

[13] DHAKATE, S.R., GUPTA, A., CHAUDHARI, A., et al., "Morphology and termal properties of PAN copolymer based electrospun nanofibers". DOI: 10.1016/j.synthmet.2010.12.019. Synthetic Metals, v. 161, pp. 411-419, Dez. 2010.

[14] SUTASINPROMPRAE, J., JITCJAICHAM, S., NITHITANAKUL, M., et al., "Preparation and characterization of ultrafine electrospun polyacrylonitrile fibers and their subsequent pyrolysis to carbon fibers".

https://doi.org/10.1002/pi.2040. Polymer International, v. 55, pp. 825-833, Mai. 2006.

[15] JUNIOR, M.S.O., RODRIGUES, B.V.M., MARCUZZO, J.S., et al., "A statistical approach to evaluate the oxidative process electrospun polyacrylonitrile ultrathin fibers". https://doi.org/10.1002/app.45458. Journal of Applied Polymer, v. 134, pp. 1-10, Jun. 2017.

[16] RWEI, S., WAY, T., CHIANG, W., et al., "Thermal analysis and melt spinnability of poly(acrylonitrile-comethyl acrylate) and poly(acrylonitrile-co-dimethyl itaconate) copolymers".

https://doi.org/10.1177/0040517517703597. Textile Research Journal, v. 0, pp. 1-12, 2017.

[17] ZHANG, L., ABOAGYE, A., KELKAR, A., et al., "A review: carbon nanofibers from electrospun polyacrylonitrile and their applications". https://doi.org/10.1007/s10853-013-7705-y. Journal of Materials Science, v. 49, pp. 463-480, Set. 2013. 
[18] GU, S.Y., REN, J., VANCSO, G.J., "Process optimization and empirical modeling for electrospun polyacrylonitrile (PAN) nanofiber precursor of carbon nanofibers”. European Polymer Journal, v. 41, pp. 2559-2568, Nov. 2005.

[19] SHOFNER, M.L., RODRIGUEZ-MACIAS, F.J., VAIDYANATHAN, R., "Single wall nanotube and vapor grown carbon fiber reinforced polymers processed by extrusion freeform fabrication".

https://doi.org/10.1016/j.compositesa.2003.07.002. Composites Part A: Applied Science and Manufacturing, v. 34, pp.1207, 2003.

[20] HOU, H., RENEKER, D.H., "Carbon nanotubes on carbon nanofibers: a novel structure based on electrospun polymer nanofibers". https://doi.org/10.1002/adma.200306205. Advanced Materials, v. 16, pp. 69-73, Jan. 2004.

[21] CHUN, I., RENEKER, D.H., FONG, H., et al., "Carbon nanofibers from polyacryloni- trile and mesophase pitch". Journal of Advanced Materials, v. 31 pp. 36-41, 1999.

[22] TAKAMI, N., SATOH, A., HARA, M., et al., "Structural and kinetic characterization of lithium intercalation into carbon anodes for secondary lithium batteries". Journal of The Electrochemical Society, v. 142, pp. 371-378, 1995.

[23] KIM, C., YANG, K.S., "Electrochemical properties of carbon nanofiber web as an electrode for supercapacitor prepared by electrospinning". https://doi.org/10.1063/1.1599963. Applied Physics Letters, v. 83, pp. 12161218, Ago. 2003.

[24] ARBIZZANI, C., MASTRAGOSTINO, M., MENEGHELLO, L., et al., "Electronically conducting polymers and activated carbon: Electrode materials in supercapacitor technology".

https://doi.org/10.1002/adma.19960080409. Advanced Materials, v.8, pp. 331-334, Abr. 1996.

[25] DUAN, X., HUANG, Y., WANG, J., et al., "Indium phosphide nanowires as building blocks for nanoscale electronic and optoelectronic devices”. https://doi.org/10.1038/35051047. Nature, v. 409, pp. 66-69, Jan. 2001.

[26] FITZER, E., FROHS, W., HEINE, M., "Optimization of Stabilization and Carbonization Treatment of PAN Fibres and Structural Characterization of Resulting Carbon Fibres". https://doi.org/10.1016/0008-

6223(86)90257-5. Carbon, v. 24, pp. 387-395, Jul. 1985.

[27] YADAV, D., AMINI, F., EHRMANN, A., "Recent advances in carbon nanofibers and their applications A review”. https://doi.org/10.1016/j.eurpolymj.2020.109963. European Polymer Journal, v. 138, pp. 109963, Ago. 2020.

[28] HAO, J., LIU, Y., LU, C., "Effect of acrylonitrile sequence distribution on the thermal stabilization reactions and carbon yields of poly(acrylonitrile-co-methyl acrylate)".

https://doi.org/10.1016/j.polymdegradstab.2017.11.010. Polymer Degradation and Stability, v. 147, pp. 89-96, Nov. 2017.

[29] BANG, Y.H., LEE, S., CHO, H.H., "Effect of Methyl Acrylate Composition on the Microstructure Changes of High Molecular Weight Polyacrylonitrile for Heat Treatment". https://doi.org/10.1002/(SICI)1097-

4628(19980627)68:13<2205::AID-APP17>3.0.CO;2-Y. Journal of Applied Polymer Science, v. 68, pp. 22052213, Out. 1997.

[30] PARK, D.U., RYU, J.H., HAN, N.K., et al., "Thermal Analysis on the Stabilization Behavior of Ternary Copolymers Based on Acrylonitrile, Methyl Acrylate and Itaconic Acid". https://doi.org/10.1007/s12221-0188782-y. Fibers and Polymers, v. 19, pp. 2439-2448, Set. 2018.

[31] FLEMING, R., PARDINI, L.C., ALVES, N., et al., "Comportamento Térmico do Copolímero de Poliacrilonitrila/Cloreto de Vinidileno". In: CONGRESSO BRASILEIRO DE ENGENHARIA E CIÊNCIAS DOS MATERIAIS, 21, 2014, Cuiabá. Anais...Cuiabá, 2014. p. 6763-6770.

[32] BRITO JÚNIOR, C.A.R., FLEMING R.R, PARDINI L.C., et al., "Análise Térmica da Poliacrilonitrila Plastificada com Glicerol em Extrusora". https://doi.org/10.1590/S0104-14282012005000055. Polímeros, v. 22, pp. 364-368, 2012.

[33] COSTA, R.G.F., OLIEVEIRA, J.E., PAULA, G.F., et al., "Eletrofiação de Polímeros em Solução. Parte I: Fundamentação Teórica". https://doi.org/10.1590/S0104-14282012005000026. Polímeros, v.22, n.2, pp.170-177, 2012.

[34] KIM, C., PARK, S., CHO. J., et al.,"Raman spectroscopic evaluation of polyacrylonitrile-based carbon nanofibers prepared by electrospinning". https://doi.org/10.1002/jrs.1233. Journal of Raman Spectroscopy, v. 35, pp. 928-933, Ago. 2004. 
[35] ZUSSMAN, E., CHEN, X., DING, W., et al.,"Mechanical and structural characterization of electrospun PAN-derived carbon nanofibers”. https://doi.org/10.1016/j.carbon.2005.03.031. Carbon, v. 43, pp. 2175-2185, Mar. 2005.

[36] OLIVEIRA, J.B., GUERRINI, L.M., OISHI, S.S., et al., "Carbon nanofibers obtained from electrospinning process”. https://iopscience.iop.org/article/10.1088/2053-1591/aaa467/meta. Materials Research Express, v.5, pp. 2-11, Fev. 2018.

[37] WANG, Y., SERRANO, S., SANTIAGO-AVILÉS, J.J., "Raman characterization of carbon nanofibers prepared using electrospinning”. https://doi.org/10.1016/S0379-6779(02)00472-1. Synthetic Metals, v. 138, pp. 423427, Out. 2002.

[38] WANG, G., PAN, C., WANG, L., et al., “Activated carbon nanofiber webs made by electrospinning for capacitive deionization”. DOI: 10.1016/j.electacta.2012.02.066. Electrochimica Acta, v. 69, pp. 65-70, Fev. 2012. [39] KOTZ, R., CARLEN, M., "Principles and applications of electrochemical capacitors". https://doi.org/10.1016/S0013-4686(00)00354-6. Electrochimica Acta, v. 45, pp. 2483-2498, Dez. 1999.

\section{ORCID}

Hugo Moreira da Silva Costa

Valdinei Euzebio Rodrigues

Jorge Tadao Matsushima

Mauricio Ribeiro Baldan

Mirabel Cerqueira Rezende

Rita Cassia Mendonça Sales Contini https://orcid.org/0000-0002-8437-2818

https://orcid.org/0000-0002-7703-1716

https://orcid.org/0000-0002-3395-3604

https://orcid.or:g/0000-0002-4465-3834

https://orcid.org/0000-0002-3735-8765

https://orcid.org/0000-0002-2160-0609 